\title{
Redes de atenção à saúde: contextualizando o debate
}

\author{
H ealth care networks: contextualizing the debate
}

Rosana Kuschnir ${ }^{1}$

Adolfo Horácio Chorny ${ }^{2}$
${ }^{1}$ Fundação O swaldo Cruz. Av. Leopoldo Bulhões 1.480/ sala 319, M anguinhos. 21041-210 Rio deJaneiro RJ.rosanak@ensp.fiocruz.br ${ }^{2}$ Departamento de Administração e Planejamento em Saúde, Escola Nacional deSaúde Pública, Fundação Oswaldo Cruz.
Abstract The proposal of organization in health services networks has been included in the broad field of integrated health care, a general denomination which includes a wide range of interventions varying in objectives and scope, which have in common the pursuit of integration strategies and tools. Among the main experiences in the field are the American integrated delivery systems and the traditional regionalized networks of the national health systems, especially after the introduction of new cooperation strategies. However, whilestrategies and organizational arrangements might be similar, context is central to debate. $\mathrm{N}$ ot considering differences concerning health systems nature may turn it difficult to grasp main distinctions in policy making which are responsible for possibilities and limitations of adopting integration strategies and tools. This article aims to look for references in the literature and international experience that might contribute to the debate on health networks building in the Brazilian Unified Health System (SUS). It discusses context regarding the creation of integrated health services networks in two cases, the British $\mathrm{N}$ ational $\mathrm{H}$ ealth Service and the American Health System, focusing specifically on the health services organization component.

Key words Health services network, Regionalization, Integrated systems, Health services organization
Resumo A proposta de organização de redes de atenção à saúde tem sido incluída no grande campo do "cuidado integrado", denominação genérica que agrupa intervenções que variam muito em objetivos e escopo, tendo em comum a busca por mecanismos einstrumentos de integração do cuidado. Entreas experiências consideradas centrais, estão os sistemas integrados americanos e as redes regionalizadas dos sistemas nacionais de saúde, especialmente após a adoção de novos mecanismos de cooperação. No entanto, ainda que possam ser observadas semel hanças nas estratégias e arranjos organizacionais, é fundamental contextualizar o debate. $\mathrm{N}$ ão consi derar a natureza distinta dos sistemas de saúde tende a obscurecer diferenças centrais do âmbito da política, que condicionam as possibilidades de aplicação em um dado sistema de instrumentos desenvolvidos em outro contexto. Este artigo tem por objetivo buscar referências na literatura e na experiência internacional que possam contribuir para 0 debate da constitui ção de redes no SUS. Busca contextualizar a discussão da organização de serviços de saúde "em redes" em dois casos, o sistema nacional de saúde britânico eo sistema privado americano, focalizando especificamente no recorte da organização de serviços.

Palavras-chave Redes de atenção, Regionalização, Sistemas integrados, O rganização de serviços 
Introdução

A organização de redes regionalizadas de serviços de saúde constituiu a premissa sobre a qual historicamente foram debatidas as propostas de reforma do sistema brasileiro - tomando como referência a experiência dos países que construíram sistemas universais e a literatura internacional -, e formou parte da bagagem do planejamento em saúde durante décadas.

No entanto, ao longo da década de noventa, pelos próprios rumos do processo de descentralização, a instituição de redes deixou de ser o eixo central em torno do qual se construía a organização de serviços, voltando ao centro do debate apenas a partir da publicação da N orma Operacional da Assistência à Saúde (N OAS) ${ }^{1}$, em 2000, eespecialmentecom o Pacto pela Saúde², em 2006.

Em seu retorno, a proposta de organização de redes tem assumido diferentes significados, utilizada de forma distinta por diferentes atores. Especificamente em seu recorte de organização de serviços de saúde, uma vertente de discussão - refletindo o debate observado na literatura e na experiência internacional -, centra-seno "campo do cuidado integrado" 3 .

Sob esta denominação genérica, é agrupada uma gama de intervenções com diferentes denominações em vários países, que variam muito em objetivos, escopo e mecanismos, que têm em comum a busca por mecanismos e instrumentos deintegração, aqui incluídos os dirigidos à prática clínica individual, à integração organizacional horizontal ou vertical e entre setores, como saúde ecuidado social ${ }^{4}$.

Entre as experiências consideradas centrais neste campo, estão os sistemas integrados americanos, uma grande variedade de arranjos organizacionais derivados da integração vertical de provedores no mercado americano e as redes regionalizadas dos sistemas nacionais de saúde, especialmente em suas recentes inovações no campo da integração do cuidado.

No entanto, ainda que possam ser observadas semelhanças nas estratégias utilizadas e nos arranjos organizacionais resultantes, focar o de bate apenas nestes aspectos, sem considerar a natureza distinta dossistemas, seus valores e princípios, tende a obscurecer diferenças centrais do âmbito da política, que condicionam, inclusive, as possibilidades de adoção dos instrumentos desenvolvidos em cada caso.

Para o sistema americano, por exemplo, não se coloca a questão da regional ização - intrinsecamente derivada dos princípios de universali- dade e equidade dos sistemas universais. Os sistemas integrados americanos são, por definição, destinados aos que podem pagar, com planos diferenciados que pressupõem acesso desigual e naturalmente não incorporam as questões da saúde pública e da ação sobre os condicionantes sociais, próprias do Estado.

Os sistemas nacionais, ao contrário, não podem abdicar de sua responsabilidade de garantia do direito e a organização de redes regionalizadas constitui-se num instrumento para ampliação do acesso e diminuição de desigualdades.

Este artigo tem por objetivo buscar referências na literatura e na experiência internacional que possam contribuir para o debate da constituição de redes no SUS. Busca contextualizar a discussão da organização de serviços de saúde "em redes" em dois casos, o sistema nacional de saúde britânico e o sistema privado americano, através da análise histórica comparada, focalizada especificamente no recorte da organização de serviços.

Redes regional izadas: o Relatório D awson e a constituição do NHS britânico

A primeira descrição completa de uma rederegionalizada foi apresentada pelo Relatório Dawson, publicado em $1920^{5}$, por solicitação do governo inglês, fruto do debate de mudanças no sistema de proteção social depois da Primeira Guerra Mundial. Sua missão era buscar, pela primeira vez, formas de organizar a provisão de serviços de saúde para toda a população de uma dada região.

Ainda hoje, a leitura do relatório surpreende por sua abrangência e profundidade. Em sua introdução, explicita que seu objetivo só poderia ser alcançado através de uma nova organização, com base na estreita coordenação entre medicina preventiva e curativa.

Para cada dado território, propõe a organização de serviços para aten ção integral à população com base formada por serviços "domiciliares" apoiados por centros de saúde primários, laboratórios, radiologia e acomodação para internação. Esta seria a "porta de entrada" do sistema, que empregaria os general practitioners (GP) - os médicos clínicos generalistas, que já então trabalhavam de forma autônoma e/ou contratados pelo sistema de seguro social.

Os centros primários, localizados em vilas, estariam ligados a centros de saúde secundários, localizados nas cidades maiores, com oferta de 
serviços especializados, cuja localização deveria se dar de acordo com a distribuição da população, os meios de transporte e os fluxos estabelecidos, variando "em tamanho e complexidade, segundo as circunstâncias".

Os casos que não pudessem ser resolvidos neste nível seriam encaminhados a um hospital de referência, ao qual os centros se vinculariam. Os profissionais trabalhariam de forma integrada, de modo que [...] o pessoal adscrito aos centros de saúde poderia acompanhar o processo em que interferiram desde o começo, familiarizar-se com 0 tratamento adotado e apreciar as necessidades do paciente depois de seu regresso ao lar.

O centro primário foi proposto como núcleo do sistema, onde os médicos generalistas poderiam se relacionar com especialistas e consultores, central ao aperfeiçoamento profissional, já que "[...] o médico sai da universidade e observa a enorme discrepância entre sua preparação e as necessidades dos pacientes que deve atender".

Para que essa coordenação fosse possível, já era apontada a necessidade de estabelecer-se um sistema uniforme de histórias clínicas; no caso de um paciente ser encaminhado de um centro a outro para fins de consulta ou tratamento, deve ser acompanhado de uma cópia de sua história clínica.

Do ponto de vista da gestão do sistema, todos os serviços - tanto curativos como preventivos estariam intimamente coordenados sob uma única autoridade de saúde para cada área. É indispensável a unidade de ideias e propósitos, assim como a comunicação completa e recíproca entre os hospitais, os centros de saúde secundários e primários e os serviços domiciliares, independentemente de que os centros estejam situados no campo ou na cidade.

Coube, portanto, ao Relatório Dawson, introduzir a territorialização, ausente atéentão dos sistemas de seguro social; apontar a necessidade de articulação entre a saúde pública - necessariamente nas mãos do Estado - e a atenção individual; e marcar a associação entre o modelo de organização de serviços e sua gestão, ao prescre ver uma autoridade desaúdeúnica no território. Do ponto de vista da organização de serviços, formulou os conceitos de níveis de atenção, porta de entrada, vínculo, referência e coordenação pela atenção primária, além de considerar os mecanismos de integração, como sistemas deinformação e de transportes.

A organização em redes foi concebida como uma resposta à questão de como garantir acesso com equidade a toda uma população. Por questões de eficiência/escala equalidade, seria necessário concentrar serviços e adotar mecanismos de referência. Para garantia deacesso ao cuidado integral, a regionalização deveria ser baseada em territórios de grande porte populacional, com autossuficiência em recursos de saúde em todos os níveis de atenção, subdivididos em distritos, sub-regiões ou microrregiões. 0 conjunto estaria sob um único comando e deveria operar de forma coordenada através de mecanismos de referência entreníveis (e/ou territórios), alimentados por sistemas de informação e de transportes.

A contraposição entre atenção primária e hospitalar não se colocava e o conceito dehierarquização referia-se à complexidade - compreendida como densidade tecnológica - e não a uma valoração maior ou menor entreosníveis. 0 primeiro nível de atenção e a assistência hospitalar eram compreen didos como el ementos indissociáveis da mesma rede, atendendo aos mesmos usuários, de acordo com a necessidade. 0 primeiro nível seria - como efetivamenteé, atéhoje - responsável pelo acesso de toda a população aos meios diagnósticos e aos serviços especializados/hospitalares.

Chama a atenção na leitura do relatório que tantas dimensões centrais à organização de sistemas, em discussão atéhoje, játenham sido abordadas em 1920. 0 relatório é também caracterizado pela flexibilidade e é enfatizado que, dentro das diretrizes estabelecidas, deveriam ser levadas em conta as condições locais, a forma como a população ocupava o território. 0 próprio esquema proposto (Figura 1) mostra a multiplicidade de relações estabelecidas entre os componentes da rede, inclusive com integração horizontal.

A proposta, no entanto, era inerentemente controversa. Sua adoção implicaria em que os hospitais filantrópicos - principais responsáveis pela atenção hospitalar à época -, desaparecessem como sistema autônomo; a ideia de organizar serviços para a cobertura de grandes territórios desafiava o conceito de governo local e os custos para a construção e manutenção dos serviços necessários seriam altos. $N$ ão se conseguiu chegar a uma proposta final e o relatório foi engavetado ${ }^{6}$.

Apenas durante a Segunda Guerra M undial, no âmbito da discussão de uma nova política de proteção social, apresentada pelo Relatório Beveridge em 1942, o relatório voltaria a servir de base à proposta de organização do novo sistema de saúde universal e equitativo.

A organização de serviços e os mecanismos de financiamento e gestão foram alvo de intenso debate e negociação. A discussão da regionalização expressava a disputa: as novas autoridades 
ção e encampação dos hospitais, as regiões foram instituídas como delegação do poder central, a partir das bases de referência de um hospital universitário, com populações da ordem de dois milhões de pessoas?

Pela relação intrínseca entre os princípios de universalidade, equidade e integralidade e a estratégia de regionalização e hierarquização, 0 modelo de organização em redes foi seguido por todos os países que construíram sistemas nacionais de saúde, como os nórdicos e o Canadá, com as devidas adaptações às especificidades locais. Preconizado pela Organização Mundial de Saúde, compõe a base da proposta dos SILOS (Sistemas Locais de Saúde), estratégia desenvolvida pela Organização Pan-Americana da Saúde a partir de meados da década de oitenta8.

Para cumprir as funções do primeiro nível, diferentes países optaram por composições distintas de recursos, como generalistas autônomos, equipes em centros de saúde ou policlínicas. M as, em todos os casos, o primeiro nível é dotado de resolutividade, dada a partir da qualificação dos recursos humanos, do acesso a meios diagnósti-

Figura 1. Esquema de organização de serviços apresentado no Relatório Dawson.

Fonte: M inisterio deSalud dela Gran Bretana ${ }^{5}$

sanitárias, responsáveis pel as regiões, seriam constituídas por um colegiado de autoridades locais ou por especial designação do poder central??

Com a vitória esmagadora do Partido TrabaIhista no pós-guerra, o $\mathrm{N}$ ational $\mathrm{H}$ ealth Service (NHS) britânico foi criado em 1948. A organização de serviços seguiu a proposta do relatório, com mudanças resultantes deacordos com a corporação médica. Como não aceitaram trabalhar como assalariados em centros de saúde, os médicos generalistas foram contratados por capitação, responsáveis por cuidados integrais à sua lista de pacientes, com grau importante de autonomia, mas mantida sua função de gate keeper, responsáveis pelas referências para os outros níveise pela manutenção do vínculo ${ }^{7}$. Uma nova forma de inserção que se mostrou muito bem-sucedida e foi mais tarde copiada por vários países.

Com relação à regionalização, considerou-se que sua construção através de colegiados com decisões tomadas por consenso havia sido inviabilizada por interesses divergentes de hospitais, autoridades locais e médicos. A pós nacionaliza- cos e terapêuticos e das articulações funcionais com os demais componentes da rede?.

Ao mesmo tempo, a construção da regionalização é acompanhada do arcabouço jurídico e dos arranjos institucionais compatíveis, seja em Estados unitários seja em países federativos, de modo a possibilitar a instituição do comando único, prescrito por Dawnson em 1920.

No caso dos Estados unitários, as regiões são estabelecidas por del egação do poder central, ainda que com um grau considerável de autonomia (desconcentração) e, no caso das federações ou de arranjos federativos - Canadá, Espanha, Itália, entre outros - , a descentralização é feita para as províncias (ou seus equivalentes). As bases para o planejamento são as regiões e os distritos eas atribuições assumidas pelos governos locais, em geral, restringem-se ao cuidado social ${ }^{10}$.

Reformas no sistema saú de britânico: da competição à integração

Inserida na ampla agenda de reforma do Estado e no questionamento de seu papel na execução de políticas, a primeira fase da reforma do sistema de saúde britânico foi implementada no início dos anos noventa, durante o governo de $M$ argareth Thatcher. Ainda que inicialmente se propusesse a modificar a base do financiamento, a resistência política à mudança dos princípi- 
os do NHS fez com que as medidas fossem focadas no aumento da eficiência no uso dos recursos públicos, no contexto de um sistema cronicamente subfinanciado ${ }^{11,12}$

A adoção do "mercado interno", com a separação das funções de financiamento e provisão e foco na competição, substituiu a provisão direta financiada por orçamentos globais. Os hospitais públicos transformados em trusts - entes públicos autônomos - passaram a disputar os contratos realizados pel as autoridades sanitárias, que poderiam comprar serviçostambém fora de suas regiões e distritos. Paralelamente, criou-se um projeto-piloto em que era transferido aos generalistas parte do orçamento da região, para que comprassem serviços para os pacientes sob sua responsabilidade, os GP's fundholders ${ }^{13}$.

Em que pesem os ganhos em eficiência em alguns campos, as medidas resultaram num grau importante de fragmentação, aumento na desigualdade de acesso eem seleção de pacientes. Os hospitais passaram a privilegiar os procedimentos mais lucrativos - em geral, cirurgias eletivas para diminuição defilas de espera - e os pacientes crônicos e idosos tornaram-se pouco atrativos; hospitais universitários, referência natural para casos mais complexos, não conseguiram garantir seus orçamentos e os custos administrativos do sistema aumentaram de forma muito significativa ${ }^{12,13}$.

O projeto fundholders foi capaz de garantir maior acesso a seus pacientes, mas criou uma clientela com acesso diferenciado, inadmissível no sistema britânico. Por outro lado, ao instituir centenas de compradores que contratavam serviços sem articulação com as autoridades regionais, contribuiu ainda mais para a fragmentação ${ }^{14}$.

O comprometimento da equidade ajudou a derrubar o governo conservador e, em 1997, os trabal histas assumiram, prometendo menos competição e mais cooperação. Durante a década seguinte, mantidos os princípios fundantes do sistema, instituiriam uma nova leva de reformas, que geraram novos arranjos para a provisão, inclusive com participação do setor privado na prestação de serviços.

$M$ antiveram a contratualização, mas com muito maior grau de controle, com definição central das diretrizes, parâmetros e indicadores para elaboração dos contratos. Transferiram a ênfase para o fortalecimento da coordenação entre os níveis da rede- ainda quebusquem manter alguma medida de competição em al guns campos - e reforçaram o papel de planejamento das autoridades sanitárias nacionais, regionais e distritais.
Foram institucionalizados alguns dos ganhos obtidos durante a primeira fase de reformas, especialmenteo aumento do escopo da atenção primária e o estímulo a arranjos cooperativos que haviam sido criados e que se tornariam a base para os Primary Care Trusts (PCT) hoje estabelecidos - grupos que congregam os generalistas e que, em conjunto com as autoridades regionais, compram serviços para seus usuários,12.

Foram também desenvolvidas estratégias de coordenação do cuidado clínico, como as clinical networks/redes clínicas para o tratamento de crônicos, articulação funcional de profissionais dos diversos níveis deatenção, organizações de pacientes esociedades de especialistas, que desenvolvem protocolos clínicos e mecanismos próprios deintegração das práticas que perpassam todos os serviços envolvidos ${ }^{15}$. 0 N HS sugeriu a possibilidade das clinical networks se transformarem em novos trusts com quem seriam realizados contratos, à semel hança dos sistemas integrados americanos $^{16}$. No entanto, ainda que a partir de 2000 tenha sido possível a integração vertical através da criação de trusts, até 2009 um número muito pequeno havia sido estabelecido e nenhum com as características de uma clinical network ${ }^{17}$.

Durante os anos 2000, a integração do cuidado - em suas várias concepções - se tornaria o foco da formulação de estratégias e de projetos-piloto empreendidos pelo N HS, com influência direta da experiência americana de sistemas integrados ${ }^{18}$.

\section{Do outro lado do Atlântico: a experiência norte-americana}

Baseado em seguro voluntário de empresas edois grandes programas públicos - o M edicare, de responsabilidade federal e dirigido à população maior de 65 anos e o M edicaid, para cobertura de populações de baixa renda, sob responsabilidade dos estados - o sistema americano é caracterizado pela segmentação e por um grau defragmentação incomparavelmente maior que os sistemas públicos, inclusive os de seguro social.

Os custos gerados pela fragmentação, aliada a formas de pagamento por itens e procedimentos, geraram o significativo crescimento do managed care, originado dos planos de pré-pagamento da década de trinta, e institucionalizados como política governamental nos anos setenta e oitenta ${ }^{11}$. Engloba diferentes arranjos organizacionais, centrados no pagamento por capitação a distintos tipos de organizações, que se respon- 
sabilizam pela provisão de todo o cuidado a um paciente, seja através de rede própria de serviços, seja por diferentes tipos de contratos estabelecidos com provedores ${ }^{19}$.

Com a transferência do risco financeiro dos pagadores aos provedores, as organizações de managed care desenvolveram mecanismos de controle de acesso e de utilização de recursos, entre os quais a instituição do generalista gatekeeper, que controla o acesso aos especialistas e a adoção muito rígida de protocolos clínicos econtrole da prática profissional ${ }^{19}$.

A fragmentação é um problema particularmente importante para o M edicare, com grande proporção de crônicos entre seus beneficiários e poucas possibilidades deinterferir na forma como se organiza a provisão ${ }^{20}$. Como alternativa, utiliza as organizações de managed care, que desenvolveram dois mecanismos principais de coordenação do cuidado a crônicos. 0 case manage ment/gerenciamento decasosédirigido a pacientes mais frágeis, identificados através de seu padrão de alta utilização de recursos. 0 coordenador, em geral enfermeiras especializadas em geriatria ou em doenças crônicas específicas, tem o papel de articular as práticas dos múltiplos provedores envolvidos no cuidado ${ }^{21}$.

Já os programas de disease management/gerenciamento de doenças crônicas específicas tem por alvo pacientes e grupos de risco. Entre seus objetivos, está o controle do processo de desenvolvimento da doença - de forma semelhante aos programas verticais - através de protocolos clínicos muito estruturados ${ }^{22}$.

As primeiras empresas especializadas em disease management foram criadas pela indústria farmacêutica - que permanece responsável por grande fatia do mercado. Do pacote comprado pelo plano, constavam os protocolos clínicos que incluíam os medicamentos providos pela própria empresa dentro do contrato ${ }^{22}$. Estes arranjos causaram estranheza e críticas entre os pesquisadores e gestores europeus, que consideraram inapropriado que em seus países os siste mas públicos garantissem fatia de mercado à indústria, ao mesmo tempo em que restringiriam a autonomia clínica dos médicos em favor dos fabricantes de medicamento ${ }^{23}$.

A partir de meados da década de noventa, os programas de disease management foram sendo ampliados em seu escopo, mudando seu foco de uma patologia específica às múltiplas necessidades de pacientes crônicos/idosos portadores de comorbidade. Ao mesmo tempo, sua adoção foi se expandindo e, sob este rótulo, hoje acomo- dam-se desde pequenas intervenções focalizadas a programas amplos em escopo e objetivos ${ }^{24}$.

Do ponto de vista da configuração do siste$\mathrm{ma}$, em resposta às formas de pagamento com cada vez mai or transferência de risco aos provedores, num mercado altamente competitivo, ainda na década de setenta, iniciou-se um intenso processo de reestruturação caracterizado pela consolidação, com a substituição dos hospitais filantrópicos que haviam sido responsáveis pela maior parte da provisão por corporações lucrativas $^{25}$.

As décadas de oitenta e noventa foram marcadas pelo movimento de integração vertical, desde entre provedores de serviços clínicos de diferentes níveis - em geral, a articulação de serviços ambulatoriais em torno de um hospital até a constituição de sistemas mais abrangentes, integrando provisão clínica, serviços de laboratórios e imagem e de produção de equipamentos e insumos ${ }^{26}$.

Este processo deu origem a diferentes combinações de provedores, com conformações estruturais muito diversas, que se tornaram conhecidas pela denominação genérica de integrated delivery systems (IDS)/sistemas integrados de provisão. A penasno período 1993-1997, foram identificados 1.917 sistemas integrados formados e 1.466 dissolvidos $^{26}$.

Os IDS foram definidos por Shortell ${ }^{27}$ como uma rede de organizações que provê, ou faz arranjos para prover, um continuum coordenado de serviços de saúdea uma população definida equeestá disposta a prestar contas por seus resultados clínicos e econômicos e pelo estado de saúde da população a que serve, definição que se tornaria a mais amplamente utilizada.

Redes em sistemas distintos: em busca de um referencial conceitual

Em que pesem as diferenças centrais entre redes regionalizadas esistemas integrados - a começar pela natureza radicalmente distinta dos sistemas de saúde nos quais estão inseridos - , a semeIhança em alguns arranjos organizacionais e na utilização de instrumentos de integração levou a um debate sobre as aproximações entre os dois modelos, que influenciou a formulação da política britânica na década de 2000 e que ajudou a conformar o grande e pouco delimitado campo dos sistemas integrados/cuidado integrado.

Em 2002, foi publicado um estudo em que o NHS era comparado à Kaiser Permanente ${ }^{28}$, uma 
organização americana de managed care de tipo grupo - que opera a maior parte dos serviços e que dispõe de seu próprio quadro de profissionais de saúde. Os resultados seriam favoráveis à Kaiser, que teria performance semelhante a custo menor. A publicação gerou polêmica e profissionais e gestores britânicos apontaram a impropriedade da comparação entre o N HS e um sistema integrado, queopera com seleção de pacientes com relação à condição econômica e idade, os principais condicionantes da utilização de serviços. Em 2004, foi publicado novo artigo ${ }^{29}$, em que eram questionadas as premissas e a metodologia utilizadas, demonstrando que os casos não haviam sido tornados comparáveis. Outros estudos se seguiram ${ }^{30}$, dando origem a um extenso debate acerca da possibilidade de aumentar a eficiência e melhorar a qualidade no NHS através da utilização dos instrumentos desenvolvidos pela Kaiser para cuidado a pacientes crônicos. Como resultado, foi instituído um programa de cooperação e troca de experiências, que apoia alguns proje tos-piloto, ainda em andamento ${ }^{31}$.

Outro experimento em integração foi desenvolvido a partir da atenção primária, utilizando o modelo americano Evercare de case management para pacientes crônicos ${ }^{17}$. $\mathrm{N}$ o entanto, não se observaram efeitos significativos nas admissões hospitalares, tempo de permanência ou mortalidade. De acordo com os estudiosos britânicos, há pouca evidência de que estas estraté gias deintegração possam reduzir internações no caso da Grã-Bretanha, já que a efetividade de abordagens complexas de case management depende do tipo específico deinterven ção, da natureza da população-alvo e das características do sistema de saúde em questão ${ }^{17}$.

Outros autores também afirmam que, dadas as diferenças entre os sistemas de saúde, os achados originados no contexto americano podem não ser facilmente transferíveis e que compreender o contexto institucional éessencial para a identificação dos facilitadores e das barreiras à integração $0^{24,32}$.

Como resultado destas experiências, o NHS assumiu uma atitude mais cautelosa, contrariamente ao que havia sido antecipado, e optou por não definir as estratégias deintegração como política nacional, lançando em abril de 2009 novos pilotos, com o objetivo de buscar mais evidências ${ }^{17}$.

No contexto deoutro sistema nacional de saúde - da Catalunha, na Espanha - , observou-se a emergência desistemas integrados deforma mais assemelhada aos arranjos verticais americanos. N este caso, a autoridade sanitária contrata uma multiplicidade de provedores de forma complementar à provisão pública financiada por orçamentos. Processos de integração vertical se deram a partir da década de noventa, quando a gestão conjunta de um hospital de agudos, um centro para tratamento de crônicos e de uma equipe de atenção primária tornou-se 0 embrião das denominadas organizações sanitárias integradas, das quais havia dezoito catalogadas em $2006^{33}$.

Portanto, no grande campo do cuidado integrado, são englobadas experiências que vão dos sistemas integrados americanos às redes regionalizadas dos sistemas nacionais de saúde, passando por um conjunto de intervenções com maior ou menor grau de abrangência em seu escopo e que podem ou não se traduzir em arranjos organizacionais de diferentes tipos. Não por acaso, do ponto de vista conceitual, éum campo pouco delimitado, sem definições comuns, com uma pletora determinologias. Foi descrito alternativamente como "um pântano acadêmico de definiçõeseanálise conceitual" ${ }^{34}$ ou maissimplesmente como uma torre de Babel ${ }^{35}$. Em revisão sistemática recente, foram encontrados mais de setenta termos ou frases relacionadas à integração, compreendendo 175 definições e conceitos ${ }^{36}$.

Nolte e McKee ${ }^{24}$ observam que a formulação deShortell refletefortementea perspectiva do managed care, em que a ênfase é numa população definida (mas selecionada), e na integração das funções de financiamento e da provisão, que nos Estados Unidos tradicional mente estiveram separadas. Esteconceito não seria facilmentecomparável com a interpretação européia, na qual tradicionalmente integração se refereà integração de setores - saúde e cuidado social - e não de funções.

Já pesquisadores ligados ao Consórcio H ospitalar da Catalunha utilizam o critério de afiliação da população para classificar os sistemas ou redes integradas em dois tipos: (1) população definida pelo território, sob gestão de uma autoridade sanitária, geralmente formando parte de um sistema nacional de saúdee (2) população definida por afiliação voluntária, nos países em que se estabeleceu um mercado para a saúde, como é o caso dos sistemas integrados americanos ${ }^{37}$.

Uma série de autores tem argumentado a necessidade de esclarecer conceitos e delimitar modelos, de modo a tornar possível a compreensão de seu significado, a troca de experiências ea avaliação de resultados ${ }^{38,39}$.

Em 2008, a Organização Pan-Americana da Saúde empreendeu consultas nacionais e regionais para validação de sua proposta de organização de redes, denominada em sua versão final 
“Redes Integradas de Serviços de Saúde Baseadas na Atenção Primária" ${ }^{3}$, que provê um marco conceitual para os países latino-americanos.

0 documento adota uma versão modificada da definição de Shortell, considerando que as Redes I ntegradas de Serviços de Saúde podem definir-se como uma rededeorganizaçõesque provê, ou faz arranjos para prover, serviços de saúde equitativos eintegraisa uma população definida e que está disposta a prestar contas por seus resultados clínicos e econômicos e pelo estado de saúde da população a que serve.

Ao basear as redes na atenção primária e introduzir o conceito deserviços de saúde equitativos e integrais, a proposta alinha-se no campo das redes regionalizadas dos sistemas públicos e forneceum marco conceitual para a organização de redes no caso brasileiro.

Uma contribuição central do documento éa clara separação entre os atributos da rede e os mecanismos e instrumentos de coordenação a serem utilizados para sua implementação. Entre os treze atributos essenciais definidos, são centraisà discussão aqui realizada: população eterritório definidos; extensa rede deestabelecimentos de saúde que presta serviços integrais; primeiro nível de atenção com cobertura de toda a população, porta de entrada do sistema, que integra e coordena a atenção e sistema de governança único para toda a rede. Estes atributos remetem à proposta de Dawson das redes regionalizadas, que assim constituídas, devem utilizar mecanismos de coordenação ao longo de todo o continuum dos serviços.

De acordo com a proposta, dada a diversidade de contextos, não seria possível prescrever um modelo organizacional único para as redese vários esquemas seriam possíveis. 0 objetivo da política pública seria o de propor um desenho que satisfaça as necessidades organizacionais específicas de cada sistema.

São identificados instrumentos de política e mecanismos institucionais, cuja pertinência dependerá de cada contexto específico. Uma questão central, ainda, é que quaisquer que sejam os mecanismos ou instrumentos utilizados, devem estar sempre respaldados por uma política de Estado que impulsione as redes como estratégia fundamental para o al cance de serviços de saúde mais acessíveis e integrais, apoiada em um referencial jurídico coerente.

\section{Conclusão}

Ao colocar no centro do debate a discussão da integração/coordenação do cuidado, o processo de reforma dos sistemas nacionais, em sua fase mais recente, passou a utilizar mecanismos einstrumentos próximos aos desenvolvidos no mercado americano. Este, por outro lado, como resposta às pressões demercado, experimentou processos de integração vertical com arranjos organizacionais em níveis de atenção, generalistas gatekeepersemecanismos de referência, quelembram os modelos tradicionais de organização em redes dos sistemas universais.

No entanto, ainda que os arranjos organizacionais e os instrumentos institucionais em busca da integração do cuidado sejam similares desenvolvidos num padrão comum de alta expectativa de vida e prevalência de doenças crônicas -, os valores, premissas e objetivos que informam cada um dos sistemas e a resultante forma de organização de serviços implicam contextos muito distintos.

Os sistemas nacionais de saúde operam redes com populações definidas geograficamente por que saúde é entendida como bem público e o sistema é universal e equitativo. As redes são o instrumento degarantia do direito, ampliando acesso e diminuindo desigualdades. A regionalização e a constituição de redes são compreendidas em suas dimensõestécnica epolítica. Ao mesmo tempo em que são a única forma de garantir acesso a cuidado integral de forma igualitária, envolvem disputas de poder erequerem decisões de política pública que certamenteferem inter esses. A região éinstitucionalizada etem comando único por que, sem institucionalidade, não é possível garantir o direito e, sem comando único, não é possível cobrar a responsabilidade sanitária.

Dependendo do contexto, esta autoridade sanitária pode utilizar a estratégia de contratualização para a garantia da provisão, inclusiveatravés de provedores privados, eaté mesmo empregar mecanismos de pagamento ou de outro tipo queincentivem a integração vertical ea emergência de sistemas integrados. No entanto, não Ihe deveria ser possível abdicar da responsabilidade sobre as condiç̧ões de saúde e o acesso da população às ações e serviços. A utilização da contratualização, pelo contrário, implica decisão política para exercício do poder regulatório, inseparável da capacidade técnica para definição do plano no qual os contratos estarão inseridos e para o desenho dos instrumentos, incluído o alinhamento dos incentivos financeiros. 
As redes regionalizadas a serem construídas no SUS claramente estão neste campo, com referencial teórico remetendo a Dawson, atualizado na proposta da OPAS. Sua construção nestes moldes, no entanto, representa alguns desafios, considerando as dimensões técnica e política, de resto indissociáveis.

Frente à peculiar combinação de uma federação trina com descentralização da responsabilidade sanitária para o nível local, como construir a regionalização? Do ponto de vista técnico, como se configuram as regiões de saúde? Qual o grau de autossuficiência da "extensa rede de serviços que provê cuidados integrais" que propõe a OPAS? Do ponto de vista político institucional, como construir o território regional? Qual o papel ea responsabilidade de cada ente federado na constituição desteespaço? Como construir a institucionalidade?

0 enfrentamento destes desafios permitirá definir um marco referencial para a constituição das redes, em suas diretrizes gerais - a ser traduzida em condições regionais específicas -, e permitiráavaliar possibilidades elimitações de modo a embasar a escolha dos mecanismos e instrumentos pertinentes.

\section{Colaboradores}

R Kuschnir eAH Chorny participaram igualmente de todas as etapas de elaboração do artigo.

\section{Referências}

1. Brasil. M inistério da Saúde. Portaria MS/GM 95, de 26/01/01. Norma Operacional de Assistência à Saúde - NOAS SUS 01/01. Diário Oficial da U nião 2001; 26 jan.

2. Brasil. M inistério da Saúde. Pactos pela Vida e de Gestão - Regulamento. Série Pactos pela Saúde 2006. Vol. 2. Brasília: M inistério da Saúde; 2006.

3. Organización Panamericana de la Salud. Redes Integradas de Servicios de Salud Basadas en la Atención Primaria.Washington, D.C.: OPAS; 2009.

4. Delnoij D, Klazinga N, Glasgow IK. Integrated care in an international perspective. Int J Integr Care [periódico na Internet] 2002 [cerca de 4 p.] Disponível em: http://www.ijic.org/index.php/ijic/article/view/ $62 / 124$

5. M inisterio de Salud de la Gran Bretana. Informe Dawson sobre el futuro de los servicios medicos y afines, 1920. Washington, D.C.: OPAS/OM S; 1964. [Publicación cientifica $n^{\circ}$ 93]

6. Webster $\mathrm{C}$. The National Health Service: a political history. Oxford: Oxford University Press; 2002.

7. Rivett CGC. From craddle to grave: 50 years of NHS. London: London King's Fund; 1998.

8. Organización Panamericana de la Salud. Los sistemas locales de salud: conceptos, métodos, experiencias. Washington, D.C: OPAS/OM S; 1990. [Publicación cientifica $n^{\circ}$ 519]

9. Rico A, Saltman R, Boerma W. Primary Health Care in the driver's seat: organizational reform in European primary care. Oxford: Oxford University Press; 2005.

10. Saltman RB, Bankauskaite V, Vrangbaek K, editors. Decentralization in $\mathrm{H}$ ealth Care: Strategies and outcomes. Berkshire: Open University Press; 2007. 
11. Almeida CM. Reforma do Estado e reforma de siste mas de saúde: experiências internacionais e tendências de mudança. Cien Saude Colet 1999; 4(2):263286.

12. Klein R. The new politics of the NHS: from creation to reinvention. Oxford: Radcliffe Publishing; 2006.

13. Saltman RB, Figueras JF, Sakellarides C, editors. Critical Challenges for $\mathrm{H}$ ealth Care Reform in Europe. London: Open University Press; 1998.

14. Wilkin D. Primary care budget holding in the United Kingdom National Service: learning from a decade of health service reform. M ed J Aust 2002; 176(9):539-542.

15. Edwards N. Clinical Networks. BMJ 2002; 324 (7329):63.

16. Department of Health. National Framework for $\mathrm{Re}$ nal Services. [site da Internet] Disponível em: http:/ /www.doh.gov.uk

17. Hawkes N. Joined up thinking. Health Policy 2009 ; 338:1238-1239.

18. Ham C. Clinically Integrated Systems: The next step in English Health Reform? [site da Internet] 2007. Disponível em: http://www.nuffieldtrust.org.uk

19. Fairfield G, Hunter DJ, M echanic D. Managed care: origins, principles and evolution. BMJ 1997; 314:1823-1833.

20. Berenson RA, Horvath J. Confronting the Barriers to Chronic $\mathrm{Care} \mathrm{M}$ anagement in $\mathrm{M}$ edicare $\mathrm{H}$ ealth $\mathrm{Af}$ fairs. [site da Internet] 2003. Disponível em: http:/ /www.content.healthaffairs.org/cgi/reprint/hlthaff. w337v1.pdf

21. Cunningham LG, Koen MJ. Improving patient care delivery with integrated case management. $\mathrm{H}$ ealth Care Financ M anag 1996; 50(12):34-45.

22. Bodenheimer T. Disease management in the American market. BMJ 2000: 320:563-566.

23. Greenhalgh $T$, Herxheimer $A$, Isaacs AJ, Beaman $M$, Morris J, Farrow S. Commercial partnerships in chronic disease management: proceeding with caution. BMJ 2000; 320:566-568.

24. Nolte $\mathrm{E}, \mathrm{M}$ CKee $\mathrm{M}$. Integration and chronic care: a review. In: Nolte $E, M c K$ ee $M$, editors. Caring for People with Chronic Conditions: A Health System Perspective. Oxford: Oxford University Press; 2008.

25. Bazzoli Gj. The corporization of American hospitals. J H ealth Polit Policy Law 2004; 294(4-5):885-905

26. Greenberg W, Goldberg LG. The determinants of hospital and HMO vertically integrated delivery systems in a competitive health care sector. Int J Health Care Finance Econ 2002; 2(1):51-68.

27. Shortell S, Gillies R, Anderson D. The new world of managed care: creating organized delivery systems. Health Affairs 1994; 13:46-54.

28. Feachem RGA, Sekhri NK, White KL. Getting more for their dollar: a comparison of the NHS with California's Kaiser Permanente. BMJ 2002; 324:135143.
29. Talbot-Smith A, Gnani S, Pollock AM, Gray DP. Questioning the claims from Kaiser. Br J Gen Pract 2004; 54(503):415-421.

30. Ham C, York N, Sutch S, Shaw R. Hospital bed utilization in the NHS, Kaiser Permanente, and the US M edicare programme: analysis of routine data. BMJ 2003; 327:1257-1260.

31. Ham C. Developing integrated care in the N HS: adapting lessons from Kaiser. [site da Internet] 2006. Disponível em: http://www.hsmc.bham.ac.uk/documents/kaiser_paper_may06.pdf

32. Mur-Veeman I. Comparing integrated care policy in Europe: Does policy matter? Health Policy 2008; 8(2):172-183.

33. Vásquez MLN, Lorenzo IV. Organizaciones sanitarias integradas. Un estudio de casos. Consorci Hospitalari de Catalunya. Barcelona: CHC; 2007.

34. Howarth $\mathrm{M}, \mathrm{H}$ aigh $\mathrm{C}$. The myth of patient centrality in integrated care: the case of back pain. Int J Integr Care [periódico na Internet] 2007 [cerca de 7 p.] Disponível em: http://www.ijic.org/index.php/ ijic/article/view/203/404

35. Kodner DL, Spreeuwenberg C. Integrated care: meaning, logic, applications, and implications - a discussion paper. Int J Integr Care [periódico na Internet] 2002 [cerca de 6 p.] Disponível em: http:/ /www.ijic.org/index.php/ijic/article/view/67

36. Armitage GD, Suter E, Oelke ND, Adair CE. Health systems integration: state of the evidence. Int J Integr Care [periódico na Internet] 2009 [cerca de 7 p.] Disponível em: http://www.ijic.org/index.php/ ijic/article/view/316/630

37. Vasques MLN, Vargas Lorenzo I. Redes integradas de servicios de salud: ¿Solución o problema? Rev. Cienc. Salud 2006; 4(1):5-9.

38. Billings, JR. What do we mean by integrated care? A European interpretation. Int J Integr Care 2005; 13(5):13-20.

39. Stein KV, Rieder A. Integrated care at the crossroads - defining the way forward Int J Integr Care [periódico na Internet] 2009 [cerca de 7 p.] Disponível em: http://www.ijic.org/index.php/ijic/article/view/315/628

Artigo apresentado em 24/05/2010

Aprovado em 02/06/2010

Versão final apresentada em 08/06/2010 